\title{
O uso da ventosaterapia como proposta de promoção à saúde para funcionários de uma universidade privada em Fortaleza - CE
}

The use of cupping therapy as a proposal to promote health for employees of a private university in Fortaleza - CE El uso de ventosaterapia como propuesta de promoción de la salud de los empleados de una universidad privada en Fortaleza - CE

\begin{abstract}
RESUMO
Objetivo: Avaliar os efeitos da ventosaterapia como forma de promoção da saúde em colaboradores de uma Universidade privada de Fortaleza-CE. Metodologia: 0 estudo foi realizado por meio de uma pesquisa intervencionista e de caráter longitudinal nos laboratórios do Curso de Estética e Cosmética da Universidade de Fortaleza, em Fortaleza- CE. O período da coleta foi de agosto a outubro de 2019, duas vezes por semana com 08 funcionários que estão alocados na Universidade, que foram convidados mediante e-mails, e pela técnica do laboratório na própria Universidade. Resultados: Verificou-se que com a terapia abordada houve uma melhora na qualidade de vida, qualidade do sono, melhora do humor, redução do uso de medicamentos para dormir e redução das dores, sintomas relatados pelos voluntários. Conclusão: 0 uso da ventosaterapia obteve diversos benefícios para a saúde dos voluntários presentes neste estudo. Entre eles, podemos destacar a melhora na qualidade do sono e consequentemente o aumento da disposição e melhora do humor, proporcionando maior qualidade de vida.
\end{abstract}

DESCRITORES: Medicina Tradicional Chinesa; Qualidade de Vida; Saúde do Trabalhador.

\section{ABSTRACT}

Objective: To evaluate the effects of cupping therapy as a way of promoting health among employees of a private University in Fortaleza-CE. Methodology: The study was carried out through an interventional and longitudinal research in the laboratories of the Course of Aesthetics and Cosmetics at the University of Fortaleza, in Fortaleza-CE. The collection period was from August to October 2019, twice a week with 08 employees who are allocated at the University, who were invited by e-mails, and by the laboratory technique at the University itself. Results: It was found that with the therapy approached there was an improvement in the quality of life, quality of sleep, improvement of mood, reduction of the use of medicines to sleep and reduction of pain, symptoms reported by the volunteers. Conclusion: The use of cupping therapy had several health benefits for the volunteers present in this study. Among them, we can highlight the improvement in the quality of sleep and consequently the increase of the disposition and improvement of the mood, providing greater quality of life.

DESCRIPTORS: Traditional Chinese Medicine; Quality of Life; Worker's Health.

\section{RESUMEN}

Objetivo: Evaluar los efectos de la eólica como forma de promover la salud entre los empleados de una Universidad privada en Fortaleza-CE. Metodología: El estudio se realizó a través de una investigación intervencionista y longitudinal en los laboratorios del Curso de Estética y Cosmética de la Universidad de Fortaleza, en Fortaleza-CE. El período de recolección fue de agosto a octubre de 2019, dos veces por semana con 08 empleados que se encuentran adscritos en la Universidad, quienes fueron invitados por correo electrónico, y por la técnica de laboratorio en la propia Universidad. Resultados: Se encontró que con la terapia abordada hubo mejoría en la calidad de vida, calidad del sueño, mejora del estado de ánimo, reducción del uso de medicamentos para dormir y reducción del dolor, síntomas reportados por los voluntarios. Conclusión: El uso de la terapia de viento tuvo varios beneficios para la salud de los voluntarios presentes en este estudio. Entre ellos, podemos destacar la mejora en la calidad del sueño y en consecuencia el aumento de la disposición y mejora del estado de ánimo, proporcionando una mayor calidad de vida. DESCRIPTORES: Medicina Tradicional China; Calidad de Vida; Salud del Trabajador.

RECEBIDO EM: 30/05/2020 APROVADO EM: 31/05/2020 


\section{Sarah Stephane Nunes Lopes}

Esteticista e Cosmetóloga pela Universidade de Fortaleza - UNIFOR.

ORCID: 0000-0003-1931-4141

\section{Vecia Maria de Souza Reis}

Esteticista e Cosmetóloga pela Universidade de Fortaleza - UNIFOR.

ORCID: 0000-0002-8361-1135

\section{Fábia Azambuja Pereira Salviano}

Fisioterapeuta. Mestre em Ensino na Saúde - UECE. Pós-graduação em Fisioterapia Dermatofuncional- Unichistus. Docente da UNIFOR.

ORCID: 0000-0003-3189-9744

\section{Cristina de Santiago Viana Falcão}

Fisioterapeuta. Mestre em Saúde Coletiva- UNIFOR. Especialista em Fisioterapia Dermatofuncional- UNIFOR. Docente da UNIFOR. ORCID: 0000-0001-9773-2301

\section{Michelli Caroline de Camargo Barboza}

Fisioterapeuta. Especialista em Fisiologia do Exercício pela Universidade Federal de São Carlos - UFSCar. Mestre em Saúde Coletiva pela Universidade de Fortaleza - UNIFOR. Doutora em Ciências da Reabilitação pela Universidade Federal de Minas Gerais - UFMG. Docente da UNIFOR.

ORCID: 0000-0003-0001-6590

\section{Aline Barbosa Teixeira Martins}

Fisioterapeuta. Doutora em Ciências Médico-Cirúrgicas - Universidade Federal do Ceará - UFC. Mestre em Saúde Coletiva UNIFOR. Especialista em Fisioterapia Dermato-Funcional - UNIFOR. Docente da UNIFOR.

ORCID:

\section{INTRODUÇÃO}

0 termo estresse indica a condição gerada pela percepção de estímulos que provocam ansiedade emocional e, ao perturbarem o equilíbrio, dão início a um processo de adaptação, sendo caracterizado pelo aumento da eliminação de adrenalina produzindo diversas manifestações no corpo, com distúrbios fisiológicos e psicológicos ${ }^{(1)}$.

No presente século, o estresse tem apresentado desequilíbrios relacionados às emoções da população(2).

Dezenas de pesquisas vêm sendo feitas de acordo com essa temática em decorrência da negatividade do estresse ocupacional na saúde e no bem-estar dos empregados, fazendo com que hajam trabalhadores estressados, diminuição do rendimento, levando ao aumento dos custos para as empresas; quando relacionada a problemas de saúde, com a elevação do absenteísmo, da rotatividade e do número de acidentes no local de trabalho ${ }^{(3)}$.

Pesquisas complementam que, o estresse ocupacional vem sendo estudado para maior entendimento da sua origem, mecanismos e consequências na saúde e desenvolvimento das atividades laborais exercidas pelos empregados ${ }^{(3,4)}$.

Um dos motivos que está vinculado às atividades mal executadas nos seus ambientes de trabalho são exatamente os excessos de cobranças, afetando o psicológico e causando estresse, gerando cansaço, dores musculares e insônia ${ }^{(5)}$.

Desse modo, as Práticas Integrativas e Complementares (PIC) vem oferecendo a capacidade de aumento da qualidade de vida de pessoas prejudicadas por inúmeras doenças, entre elas o estresse. Com a aplicação de diversas terapias, obtém-se melhora na saúde desses indivíduos. Algumas das que podemos citar são: a homeopatia, a fitoterapia, a imunoterapia, o toque terapêutico, o Reiki, o desbloqueio/alinhamento de chacras, a terapia artística, a eurritmia curativa, a visualização, a troca de convivência em grupo, a meditação, o trabalho biográfico antroposófico e o relaxamento através da ventosaterapia ${ }^{(6)}$.

A ventosaterapia tem sido um procedimento muito procurado para quem busca a estética como forma de conforto e saúde, tendo em vista que esse método de tratamento procede em duas áreas, trazendo ótimas respostas terapêuticas, proporcionando ampliação na circulação e oxigenação dos tecidos mediante uma massagem ${ }^{(7)}$.

Para manter a saúde, é necessário cuidar não somente do corpo como também da alma e espírito. Diante de tantos benefícios que essa técnica pode nos trazer, têm-se destacado o seu uso para tratar pessoas afetadas nos ambientes de trabalho ${ }^{(8)}$.

Estudos recentes apontam a importância da ventosaterapia como uma forma terapêutica em inúmeras condições clínicas, sendo definida como uma terapia complementar que consiste basicamente em aplicar um vácuo em alguns pontos da pele utilizando copos em áreas que possuem dor, pontos de acupuntura ou zonas de reflexo. $\mathrm{O}$ vácuo formado pelo copo faz com que a microcirculação da pele libere toxinas aumentando a circulação local ${ }^{(9)}$.

$\mathrm{O}$ tratamento com ventosaterapia tem a finalidade de atingir o equilíbrio energético, tendo em vista que essas patologias ocorrem, na maior parte dos casos, por fa- 
tores emocionais. A aplicação das ventosas nos pontos Shu dorsais, que representam os pontos de assentimento dos Zang-Fu, trará benefícios para os pacientes, considerando que as ventosas agem retirando o excesso de energia dos Zang-Fus estagnados, fazendo com que o sangue acometido possa circular, diminuindo o calor e as energias ruins, assim, restabelecendo o equilíbrio ${ }^{(10)}$.

O uso de terapias holísticas é pouco conhecido como terapias de relaxamento, contudo, estudos apontam a ventosaterapia como uma terapia extremamente eficaz na diminuição de dores, tensões musculares, e aumento da qualidade de vida de pessoas que passam por um alto teor de estresse e uma intensa carga horária de trabalho. Esse tema foi escolhido com intuito de melhorar da qualidade de vida em colaboradores da universidade.

Este estudo tem como objetivo verificar os efeitos da ventosaterapia como proposta de promoção à saúde para funcionários de uma universidade privada em Fortaleza-CE.

\section{METODOLOGIA}

Tratou-se de um estudo quantitativo, intervencionista e de caráter longitudinal, realizado nos laboratórios do Curso de Estética e Cosmética da Universidade de Fortaleza, em Fortaleza-Ceará. O período da coleta foi de agosto a outubro de 2019, uma vez por semana, totalizando 12 atendimentos. A amostra constou de 08 funcionários, que foram convidados mediante e-mails e cartazes colocados nos diversos setores da universidade.

Foram incluídos na pesquisa, homens e mulheres entre 25 e 65 anos, contratados há pelo menos um ano. Foram excluídos do estudo funcionários com alguns problemas de saúde e de acordo com as contra indicações da ventosaterapia, que são: fragilidade capilar, câncer, feridas abertas, inflamações e febre.

Os voluntários foram inicialmente avaliados e submetidos a uma ficha de avaliação para identificar a inclusão na pesquisa. Esclarecemos que não é possível aplicar as ventosas todas as vezes no mesmo local, devido à precisão de um tempo mínimo para que o sistema circulatório cumpra com sua função de aumento da oxigenação até as manchas desaparecem por inteiro.
Os participantes responderam ao QWLQ 78 , proposto por Junior, Pilatti, Pedroso ${ }^{(11)}$, que é um questionário específico validado sobre qualidade de vida no trabalho, através de um teste de autopreenchimento com perguntas para serem respondidas segundo uma escala de 1 a 5 ( 1 - nada, 2 - muito pouco, 3 - mais ou menos, 4 - bastante, 5 - extremamente).

Todos os pacientes assinaram o Termo de Consentimento Livre Esclarecido (TCLE) denotando concordância com a metodologia aplicada e etapas do estudo.

$\mathrm{Na}$ anamnese, todos os pacientes relataram sentir maior desconforto na região do trapézio e lombar. Desta forma, os voluntários foram posicionados em decúbito ventral, realizou-se uma higienização das costas com um fluído corporal. Em seguida, aplicou-se o creme de massagem corporal em toda a região, fazendo deslizamento com as polpas digitais em forma de massagem a fim de identificar os locais com pontos de tensões. Após, aplicou-se o copo de acrílico, característico da ventosaterapia, deslizando por toda a extensão das costas, fazendo 05 tipos de movimentos, até hiperemiar. A pressão aplicada foi variada, de acordo com as características individuais de cada voluntário e baseado na anamnese.

A aplicação da ventosa de forma deslizante e fixa não apresentou manchas, como hematomas. Ocorreu modificação na coloração local, algumas avermelhadas, marrons e roxas, não foi observada coloração preta. Essas observações serviram como análise da qualidade do sangue $^{(12)}$.

Com isso, a pele foi purificada através da captação de maior quantidade de oxigênio, o que possibilitou aumento dos movimentos de fluidos naturais. A pressão negativa foi utilizada com o objetivo de limpar o sangue paralisado ${ }^{(13)}$.

Posteriormente, posicionou-se a ventosa de maneira fixa, seguindo os pontos de acupuntura, que devem permanecer sobre a pele do paciente, variando de cinco a dez minutos de maneira suficiente para se obtiver os resultados desejados ${ }^{(14)}$.

Nas primeiras três sessões, os copos permaneceram por sete minutos e nas sessões seguintes, dez minutos. Os copos de ventosaterapia eram posicionados na região paravertebral e de acordo com a individu- alidade de cada voluntário, baseando-se em seu maior ponto de tensão. Quando necessário, eram aplicados junto ao copo os pinos nos pontos gatilho. Ao retirar esses objetos do corpo, finalizou-se com um deslizamento com creme de massagem no local para amenizar a vermelhidão e explicou-se aos pacientes que tais manchas iriam desaparecer após alguns dias e que não deveriam se expor ao sol, mas. caso fosse necessário. que fizesse o uso do filtro solar, a fim das manchas não se tornarem prolongadas ou até mesmo definitivas.

Os dados foram analisados de forma quantitativa através do uso de gráficos com auxílio do programa Excel, a fim de apresentar os resultados gerais. Já no que diz respeito às perguntas referentes às questóes, utilizando questionamento acerca das mudanças em sua rotina diária relacionadas à melhoria $\mathrm{da}$ qualidade de vida, através do Questionário de avaliação da qualidade de vida no trabalho QWLQ 78. Os dados qualitativos serviram como dados ilustrativos dos voluntários, a fim de mensurar o grau de bem-estar e satisfação com a terapia proposta.

O estudo foi aprovado pelo Comitê de Ética da Universidade de Fortaleza (CEP/ UNIFOR) com o número 3.711.345.

\section{RESULTADOS}

A amostra contou com a participação de $62,5 \%(n=5)$ mulheres e $37,3 \%$ de $(n=3)$ homens, na faixa etária de 25 a 65 anos, com profissões variadas, sendo 3 secretárias, 2 professoras, 1 técnica de laboratório, e 1 assistente administrativo. Com relação à queixa principal, todos os voluntários, $100 \%,(\mathrm{n}=8)$ responderam apresentar dor na região de trapézio e lombar. Todos os voluntários, $100 \%$, $(n=8)$ negaram a presença de alergias, feridas abertas, acidente vascular cerebral ou algum distúrbio hemorrágico, febre, neoplasias, fraturas no local a ser tratado, hepatite, doenças crônicas, infecção, sensibilidade tecidual alterada, fragilidade capilar e equimoses. Vinte e cinco por cento $(n=2)$ apresentou cirurgia anterior, especificamente cesariana. Uma das voluntárias, $12,5 \%(\mathrm{n}=1)$ relatou que já havia feito atendimentos de ventosaterapia previamente por 2 meses. 


\section{artigo}

Lopes, S.S.N.; Reis, V.M.S.; Salviano, F.A.P.; Falcão, C.S.V.; Barboza, M.C.C.; Martins, A.B.T.

O uso da ventosaterapia como proposta de promoção à saúde para funcionários de uma universidade privada em Fortaleza - CE

Baseando-se na avaliação do questionário QWLQ78, que diz respeito à avaliação da qualidade de vida no trabalho, observou-se na pergunta 1 que diz respeito a como você avalia a sua liberdade para criar coisas novas no trabalho. Notificou-se que após os atendimentos, $25 \%(\mathrm{n}=2)$ aumentou a sua liberdade de criação no trabalho, para $37,3(n=3)$ permanece a mesma e para $37,5(n=3)$ essa liberdade diminuiu.

No Gráfico 1, referente à pergunta 2, relacionada à medida que você avalia sua motivação para trabalhar, notou-se que $25 \%(\mathrm{n}=2)$ aumentou a motivação para trabalhar, $50 \%(n=4)$ persiste a mesma e em 25\% ( $n=2)$ essa motivação diminuiu.

$\mathrm{Na}$ pergunta 3 , que faz jus a como você avalia a igualdade de tratamento entre os funcionários $12,5 \%(\mathrm{n}=1)$ afirmou que essa igualdade no tratamento entre eles subiu, $62,5 \%(n=5)$ declarou a permanência do tratamento e $25 \%(\mathrm{n}=2)$ afirmou a diminuição da igualdade de tratamento.

No Gráfico 2 que explana sobre a pergunta $4 \mathrm{e}$ diz respeito à medida que você avalia o seu sono, verificou-se que $87 \%(n=7)$ relatou o aumento da qualidade do sono e $12,5 \%$ $(\mathrm{n}=1)$ declarou que a avaliação do seu sono continua a mesma.

Na pergunta 5 que faz jus a como você ava-

Gráfico 1. Em que medida você avalia sua motivação para trabalhar? Fortaleza, CE, Brasil, 2019 ( $n=8)$

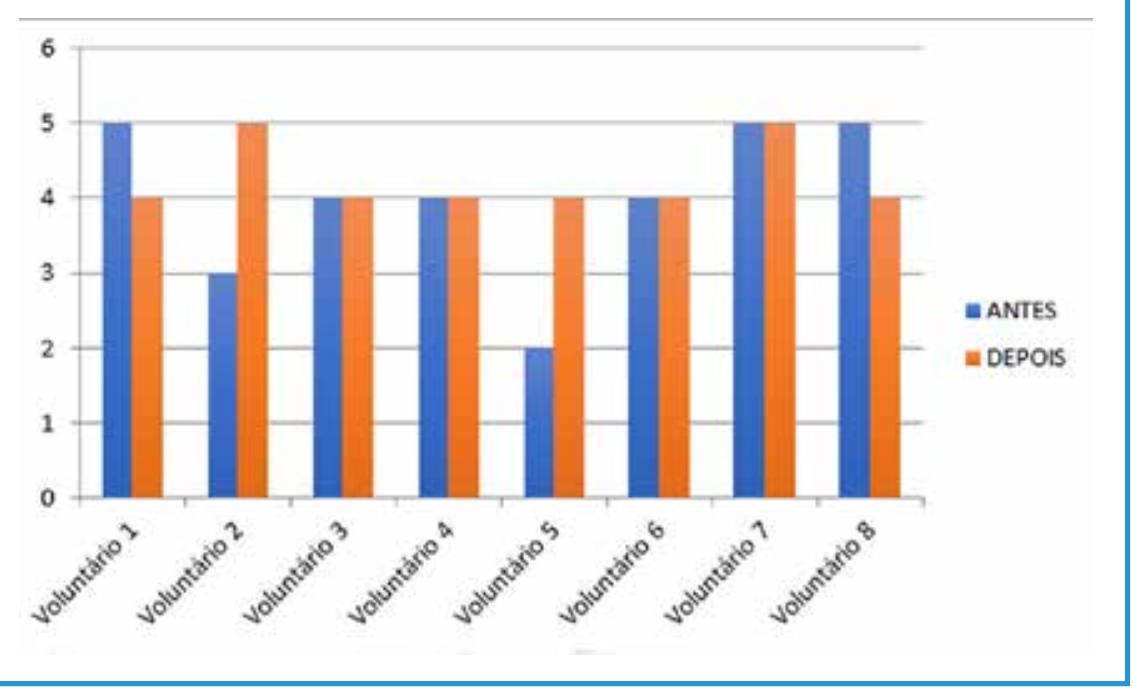

Gráfico 2. Em que medida você avalia o seu sono? Fortaleza, CE, Brasil, 2019 (n=8)

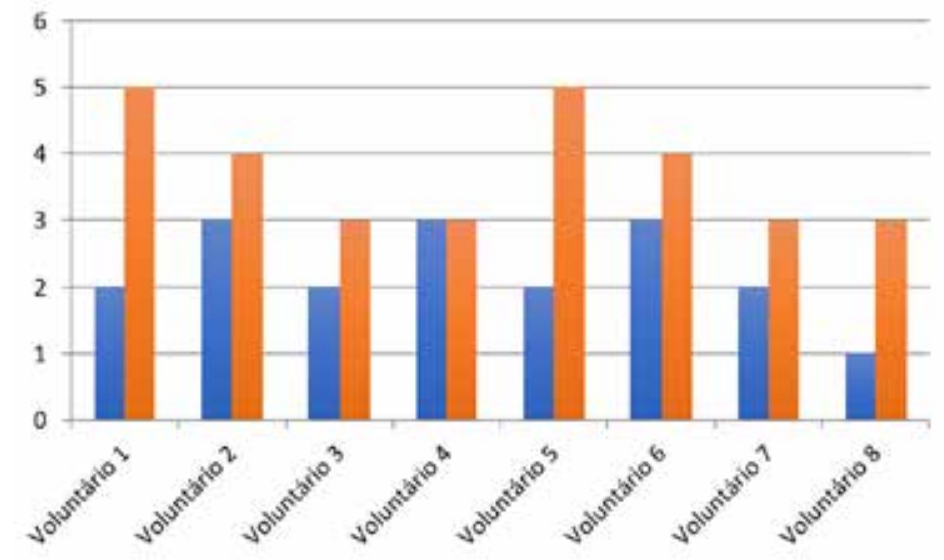

- ANTES $=$ DEPOIS lia a sua liberdade de expressão no trabalho, $37,5 \%(\mathrm{n}=3)$ notou um aumento na liberdade de expressão, $37,5 \%$ permanece a mesma e $25 \%$ afirma ter diminuído.

A pergunta 6 que é sobre sua realização com o trabalho que faz, $37,5 \%(\mathrm{n}=3)$ aumentou essa realização, $12,5 \%(\mathrm{n}=1)$ permaneceu com a mesma realização e $50 \%(n=4)$ sentiu-se mais realizada com o exercício do trabalho.

A pergunta 7 retrata o quanto você possui orgulho da organização na qual trabalha, sendo analisado que, $50 \%(n=4)$ aumentou este orgulho pela organização e $50 \%(n=4)$ permaneceu com a mesma opinião.

O Gráfico 3 é referente a pergunta 8 e fala sobre medida que algum problema com o sono prejudica seu trabalho, verificou-se que $37,5 \%(n=3)$ afirmou um aumento prejudicial no trabalho referente ao sono, $37,5(\mathrm{n}=3)$ permaneceu com a mesma opinião e $25 \%$ $(n=2)$ disse ter diminuído essa relação.

A pergunta 9 refere-se à medida em que você sente orgulho pela sua profissão, observou-se que $25 \%(\mathrm{n}=2)$ teve um aumento desse orgulho, $62,5(\mathrm{n}=5)$ permaneceu e $12,5 \%$ $9 n=1$ ) diminuiu esse orgulho pela profissão.

Constatou-se que na pergunta 10 , sobre como você avalia a qualidade da sua relação com os seus superiores e/ou subordinados que, $12,5 \%(\mathrm{n}=1)$ aumentou a qualidade $\mathrm{da}$ relação com os subordinados e/ou superiores, $62,5(n=5)$ permaneceu a mesma opinião e $25 \%(n=2)$ diminuiu a qualidade da relação.

Observou-se na pergunta 11 que faz jus à medida que sua família avalia o seu trabalho que, $50 \%(\mathrm{n}=4)$ aumentou sua opinião e $50 \%$ $(n=4)$ permaneceu com a mesma.

Verificou-se na pergunta 12 que fala sobre em que medida você está satisfeito com seu nível de participação nas decisões da empresa que, $25 \%(\mathrm{n}=2)$ está mais satisfeito, $37,5 \%$ $(n=3)$ continuou da mesma forma e $37,5 \%$ $(\mathrm{n}=3)$ diminuiu essa satisfação com o nível de participação nas decisões.

Constatou-se na pergunta 13 que analisa se você está satisfeito com o seu nível de responsabilidade no trabalho que, $50 \%(n=4)$ permaneceu com a mesma satisfação e 50\% ( $\mathrm{n}=4)$ diminuiu esse nível.

Observou-se na pergunta 14, que diz respeito à satisfação com os treinamentos dado pela organização que, $37,5 \%(n=3)$ está mais 
satisfeito, $50 \%(\mathrm{n}=4)$ permaneceu com o mesmo nível e $12,5 \%(n=1)$ reduziu a satisfação.

A pergunta 15 fala sobre da medida que você é respeitado pelos seus colegas e superiores, nela notou-se que $25 \%(\mathrm{n}=2)$ sente-se mais respeitado, $62,5 \%$ permanece com a mesma opinião e $12,5 \%$ reduziu à medida que são respeitados pelos colegas e superiores.

Verificou-se, na pergunta 16 que analisa se você se sente satisfeito com a variedade das tarefas que realiza que, $25 \%$ $(\mathrm{n}=2)$ sentiu-se mais satisfeito com a variedade de tarefas, $50 \%(n=4)$ permaneceu com a mesma satisfação e $25 \%(n=2)$ está menos satisfeito.

A pergunta 17 aborda a seguinte questão: Suas necessidades fisiológicas básicas são satisfeitas adequadamente? Nela, constatou-se que $25 \%(\mathrm{n}=2)$ está mais satisfeito, $25 \%(\mathrm{n}=2)$ permaneceu com o mesmo grau de satisfação e $50 \%(n=4)$ diminuiu a satisfação com relação às necessidades fisiológicas básicas.

$\mathrm{Na}$ pergunta 18 , que diz respeito a como você avalia o espírito de camaradagem na sua empresa, comprovou-se que $25 \%(\mathrm{n}=2)$ avaliou melhor o espírito de camaradagem e $75 \%$ $(n=6)$ permaneceu com a mesma opinião.

Referente à pergunta 19 , que verifica à medida que você se sente confortável no ambiente de trabalho. Vinte e cinco por cento $(\mathrm{n}=2)$ está mais confortável no ambiente de trabalho, $50 \%(n=4)$ permanece com o mesmo grau de satisfação e $25 \%$ $(\mathrm{n}=2)$ está menos satisfeito.

A pergunta 20, explanada no Gráfico 4, é sobre o quanto você é satisfeito com a sua qualidade de vida no trabalho. Nela, $25 \%(n=2)$ está mais satisfeito, $60 \%(n=5)$ permaneceu com a mesma satisfação com
Gráfico 3. Em que medida algum problema com o sono prejudica o seu trabalho? Fortaleza, CE, Brasil, 2019 ( $n=8)$

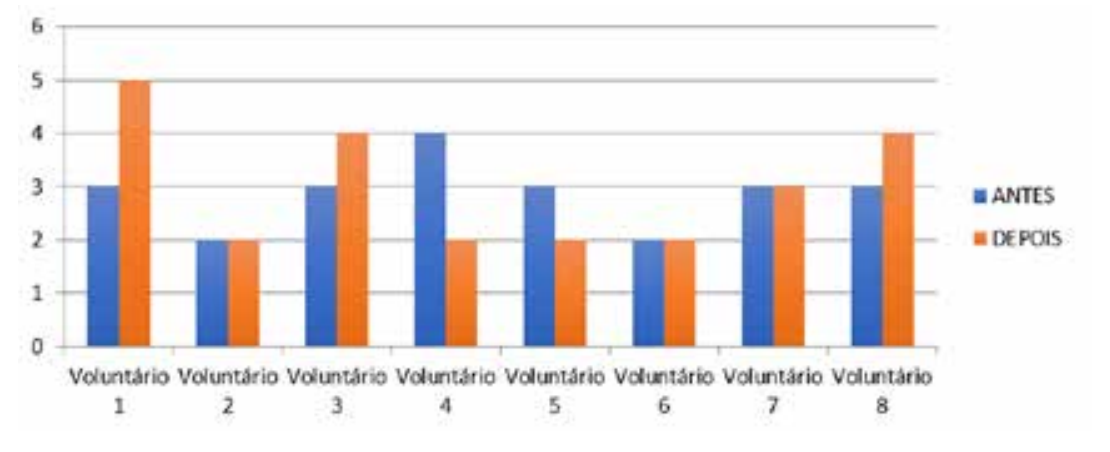

Gráfico 4. Em que medida você se sente confortável no ambiente de trabalho? Fortaleza, CE, Brasil, 2019 ( $n=8$ )

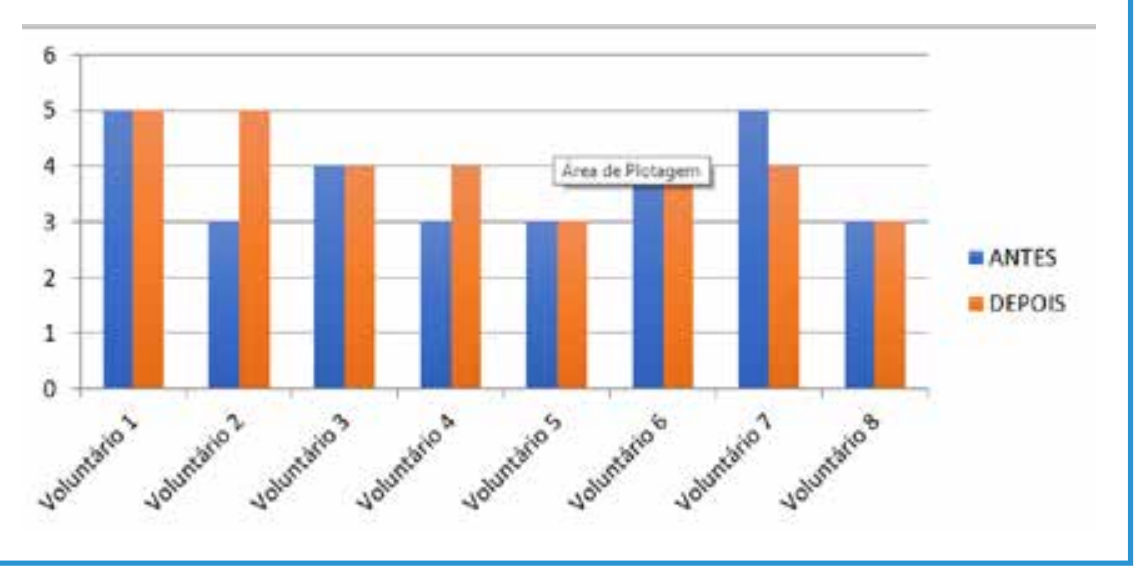

a qualidade de vida no trabalho e $12,5 \%$ $(\mathrm{n}=1)$ diminuiu a satisfação.

Após a aplicação do questionário QWLQ-(modificado), os voluntários também foram avaliados mediante uma avaliação individualizada, em que foram convidados a responder algumas perguntas. Quando questionados acerca de "Faça um breve resumo da sua experiência com a Ventosaterapia”, segue abaixo as falas de alguns voluntários:

\section{"Melhorou as dores e diminuiu a quantidade de medicamentos para alivio de dores e melhorou muito $o$ sono" (V.M.C.O., 61 anos).}

"Foi muito satisfatório, obtive bastante resultados com o tratamento, as alunas eram totalmente profissionais e eu pretendo continuar fazendo esse tratamento" (B.L.O., 25 anos).

\section{"O tratamento de ventosa trouxe uma melhora imediata na qualidade de vida, houve um aumento da disposi- ção e melhoria das dores" (M. P. S. P., 27 anos).}

"Tive uma ótima experiência, foi notável a melhoria das dores que diminuiram e durante o tempo que eu fazia as sessões minha postura melhorou muito" (A.B., 43 anos).

"Através do tratamento com ventosaterapia pude trabalhar nas tensões que me incomodavam durante o dia e a noite. Notei que com o tratamento as dores diminuiram e a disposição e o humor aumentaram, proporcionando uma qualidade de vida melhor" (I.S.M., 27 anos).

\section{DISCUSSÃO}

Nesse estudo, a ventosaterapia mostrou-se eficaz no combate à dor nas costas, em homens e mulheres adultos, não somente em variáveis relacionadas à dor, como também em melhora relacionada à qualidade do sono, aumento da disposição no ambiente de trabalho, maior produtivi- 


\section{artigo}

Lopes, S.S.N.; Reis, V.M.S.; Salviano, F.A.P.; Falcão, C.S.V.; Barboza, M.C.C.; Martins, A.B.T.

O uso da ventosaterapia como proposta de promoção à saúde para funcionários de uma universidade privada em Fortaleza - CE

dade nas atividades realizadas no trabalho, aumento da disposição para a prática de atividade física e melhora do humor.

Moura et al ${ }^{(15)}$ realizaram um estudo através de uma revisão sistemática da literatura para analisar a intensidade da dor nas costas de clientela adulta. Elaborou perguntas norteadoras para analisar a dor crônica nas costas com adultos que tinham 18 anos ou mais, que relatavam sentir esse incômodo há mais de três meses em pelo menos um dos segmentos da coluna vertebral, cervical, torácica e/ou lombar. Após análise e realização da técnica com diversas formas de aplicação, viu-se que de uma forma ampla, os resultados mostraram variação substancial na aplicação da ventosaterapia. Esses achados corroboraram com este estudo, em que os voluntários avaliados também apre- sentaram melhora na dor nas costas com a aplicação da ventosaterapia.

Freire e Peixoto ${ }^{(16)}$ realizaram estudo dos efeitos da aplicação da ventosaterapia na dor lombar crônica por meio de sucção da pele com a ventosa associada ao calor com intenção de alcançar o resultado da liberação miofascial com a utilização de água morna com a técnica de varredura, utilizando pressão de acordo com cada participante e respeitando o grau de tolerância de dor de cada um deles, permanecendo durante 10 minutos e observando o aumento do fluxo sanguíneo da região. Verificou-se que, depois de aplicada a ventosa, houve a melhora significativa da capacidade e qualidade de vida, aumentou a amplitude do movimento e reduziu a dor lombar em todos os participantes de forma homogenia.

\section{CONSIDERAÇÕES FINAIS}

$\mathrm{O}$ uso da ventosaterapia obteve diversos beneficios para a saúde dos voluntários presentes neste estudo, dentre eles, podemos destacar a melhora na qualidade do sono e, consequentemente, o aumento da disposição e melhora do humor, proporcionando maior qualidade de vida.

Todos os participantes relataram ter relaxado durante as sessões de tratamento e observaram que a técnica de ventosaterapia é benéfica no que diz respeito à redução de tensão muscular. Também houve a percepção de que, após concluir a terapia, um dos participantes reduziu a quantidade de medicamentos via oral, por conta do alívio de dores localizadas.

Com isso, notamos que a técnica utilizada é benéfica e eficazem diversas enfermidades, sejam relacionadas ao fator emocional ou fisico. -

\section{REFERÊNCIAS}

1. Croda JHR, Garcia LP. Resposta imediata da Vigilância em Saúde à epidemia da COVID-19. Epidemiol serv saúde. 2020;e2020002-e2020002.

2. Souza WM et al. Epidemiological and clinical characteristics of the COVID-19 epidemic in Brazil. Nature Human Behaviour. ago. 2020; (4):856-865.

3. OPAS. Organização Panamericana de Saúde. OPAS/OMS Brasil - Folha informativa - COVID-19 (doença causada pelo novo coronavírus) | OPAS/OMS [Internet]. Pan American Health Organization / World Health Organization. 2020 [citado 9 de junho de 2020]. Disponível em: https://www.paho.org/bra/index.php?option=com_content\&view=article\&id=6101: covid19\&ltemid $=875$

4. Brasil. Ministério da Saúde. Secretaria de Vigilância em Saúde. Boletim Epidemiológico Especial: Doença pelo Coronavírus COVID-19 - Semana Epidemiológica 27 (28/06 a 07/07). Brasília; 2020 p. 59.

5. Brasil. Ministério da Saúde. Boletim Epidemiológico Especial 16: COE-COVID 19/ Semana Epidemiológica 21 (17 a 23/05). 2020. Report No.: 16.

6. Lopes K. Bares e restaurantes têm autorização para reabertura a partir de 8 de junho em Cuiabá: Estabelecimentos devem cumprir as medidas de segurança necessárias para evitar a proliferação do novo coronavírus, que já matou 49 pessoas no estado [Internet]. 2020 [citado 9 de junho de 2020]. Disponivel em https://g1.globo.com/mt/mato-grosso/noticia/2020/05/28/ bares-e-restaurante-tem-autorizacao-para-reabertura-a-partir-de-8-de-junho-em-cuiaba.ghtml.

7. Brasil. Ministério da Saúde. Coronavírus Brasil [Internet]. 2020 [citado 13 de julho de 2020]. Disponivel em: https://covid.saude.gov.br/

8. Brasil. População residente: Censos (1980, 1991, 2000 e
2010), Contagem (1996) e projeções intercensitárias (1981 a 2012), segundo faixa etária, sexo e situação de domicílio [Internet]. 2020 [citado 8 de junho de 2020]. Disponível em: https:// datasus.saude.gov.br/populacao-residente/

9. ARPEN-BRASIL. Associação Nacional dos Registradores de Pessoas Naturais. Especial COVID-19: Óbitos com suspeita ou confirmação de COVID-19 por sexo e faixa etária [Internet]. 2020 [citado 9 de junho de 2020]. Disponivel em: https://transparencia.registrocivil.org.br/especial-covid

10. Rozenberg S, Vandromme J, Martin C. Are we equal in adversity? Does Covid-19 affect women and men differently? Maturitas. agosto de 2020;138:62-8.

11. Lima DLF, Dias AA, Rabelo RS, Cruz ID da, Costa SC, Nigri FMN, et al. COVID-19 no Estado do Ceará: Comportamentos e crenças na chegada da pandemia. Ciência \& Saúde Coletiva. 2020;25(5):1575-86.

12. Brasil. Ministério da Saúde. Boletim Epidemiológico Especial 17: COE - COVID 19/ Semana Epidemiológica 21 (17 a 23/05). 2020. Report No.: 17.

13. Carvalho T. UTIs estão prontas, mas paradas por falta de profissionais, revela Mendes [Internet]. Estadão Mato Grosso. 2020 [citado 15 de julho de 2020]. Disponivel em: https://estadaomatogrosso.com.br/politica/utis-estao-prontas-mas-paradas-por-falta-de-profissionais-revela-mendes/8442

14. SES/MT. Secretaria de Estado de Saúde, MT. Boletim Informativo No 122: Situação epidemiológica SRAG e COVID-19. 2020. Report No.: 122.

15. Shinkai S, Konishi M, Shephard RJ. Aging and immune response to exercise. Can J Physiol Pharmacol. maio de 1998;76(5):562-72. 